\title{
Soluble guanylyl cyclase regulates skeletal muscle fiber type plasticity, fatigue resistance and whole body insulin resistance
}

\author{
Younghye Moon ${ }^{1}$, Jordan Balke ${ }^{1}$, Michael Siegel ${ }^{2}$, Peter Brouckaert ${ }^{3}$, Emmanuel S Buys ${ }^{4}$, David Marcinek ${ }^{2,5}$, \\ Justin Percival ${ }^{1 *}$
}

From 7th International Conference on cGMP Generators, Effectors and Therapeutic Implications

Trier, Germany. 19-21 June 2015

Pathogenic defects in NO-cGMP signaling drive skeletal muscle dysfunction in Duchenne muscular dystrophy (DMD). These defects arise from decreased nNOS and guanylyl cyclase (GC) activity and loss of spatial control of NO production. Therapeutics such as sildenafil that amplify NO-cGMP signaling reduce skeletal muscle dysfunction in DMD patients and mouse models of DMD making GC and cGMP attractive therapeutic targets. However, GC and cGMP functions in skeletal muscle are poorly defined hindering therapy development. To remove this barrier, we investigated the functions of GC in skeletal muscle.

We report that $\alpha 1 \beta 1$ soluble GC (sGC) is the primary cGMP source in skeletal muscle. $\alpha 1 \beta 1$ sGC expression was greater in oxidative muscles suggesting a greater cGMP synthesis capacity and muscle-specific differences in sGC function. Interestingly, sGC activity exhibited partial nNOS dependence. Analyses of sGC subcellular localization revealed a pool of $\alpha 1 \beta 1$ at the cis-Golgi complex in muscle cells. $\alpha 1 \beta 1$ and $\alpha 2 \beta 1$ sGC localized to the microvasculature. Muscles lacking a functional $\alpha 1 \mathrm{sGC}$ subunit ( $\alpha 1 \beta 1$ sGC deficient) exhibited reduced fatigue resistance and normal hypertrophic growth. Surprisingly, $\alpha 1 \beta 1$ sGC deficiency had no impact on mitochondrial content suggesting that mitochondrial density may not be as tightly regulated by cGMP as previously thought. $\alpha 1 \beta 1$ sGC deficiency had a modest effect on mitochondrial ATP synthesis. Also, loss of $\alpha 1 \beta 1$ sGC triggered a type IIA to type IIX fiber shift. Although this shift was unlikely to significantly enhance fatigability, it may impact insulin metabolism because type IIX fiber content positively correlates

\footnotetext{
* Correspondence: j.percival@med.miami.edu

'Department of Molecular and Cellular Pharmacology, University of Miami Miller School of Medicine, Miami, FL 33136, USA

Full list of author information is available at the end of the article
}

with insulin insensitivity. Indeed, $\alpha 1 \beta 1 \mathrm{sGC}$ null mice exhibited gender-specific defects in whole body insulin sensitivity consistent with reports of gender-specific effects of cGMP on metabolism but normal glucose tolerance indicating compensatory changes in glucose metabolism.

In summary, these findings argue that NO signaling through sGC plays important roles in muscle fatigue and fiber type specification and suggest sGC as a target for mitigating skeletal muscle fatigue in DMD. These data also suggest that disruption of NO-cGMP signaling may contribute to the poorly understood metabolic defects in DMD patients. By showing that reductions in cGMP synthesis promote muscle fatigue, type IIX content and insulin resistance, these data suggest new insights as to how decreases in cGMP may contribute to the development of metabolic dysfunction and disease, particularly over time. Importantly, these results suggest sGC as a potential target for mitigating insulin resistance.

\begin{abstract}
Authors' details
${ }^{1}$ Department of Molecular and Cellular Pharmacology, University of Miami Miller School of Medicine, Miami, FL 33136, USA. ${ }^{2}$ Department of

Bioengineering, University of Washington, Seattle, WA 98109, USA. ${ }^{3}$ Department for Molecular Biomedical Research and Biomedical Molecular Biology, Ghent University, B-9052 Ghent, Belgium. ${ }^{4}$ Department of Anesthesia, Critical Care and Pain Medicine, Anesthesia Center for Critical Care Research, Massachusetts General Hospital, Boston, MA 02114, USA. ${ }^{5}$ Department of Radiology, University of Washington, Seattle, WA 98109, USA.
\end{abstract}

Published: 2 September 2015

doi:10.1186/2050-6511-16-S1-A8

Cite this article as: Moon et al:: Soluble guanylyl cyclase regulates

skeletal muscle fiber type plasticity, fatigue resistance and whole body insulin resistance. BMC Pharmacology and Toxicology 2015 16(Suppl 1):A8. 